\title{
Transition écologique et trajectoires sociales : les traducteurs de la mobilité durable à Mexico entre expertise internationale et politique locale \\ Ecological transition and social trajectories: the translators of sustainable mobility in Mexico City between international expertise and local politics
}

\author{
Audrey Chérubin
}

Numéro 82, 2019

Engagements et action publique face à la crise climatique

URI : https://id.erudit.org/iderudit/1061882ar

DOI : https://doi.org/10.7202/1061882ar

Aller au sommaire du numéro

\section{Éditeur(s)}

Lien social et Politiques

ISSN

1703-9665 (numérique)

Découvrir la revue

Citer cet article

Chérubin, A. (2019). Transition écologique et trajectoires sociales : les traducteurs de la mobilité durable à Mexico entre expertise internationale et politique locale. Lien social et Politiques, (82), 181-201.

https://doi.org/10.7202/1061882ar

\section{Résumé de l'article}

Cet article cherche à comprendre les ressorts de la « transition » vers une mobilité urbaine durable engagée depuis le début des années 2000 dans la ville de Mexico, en dépit d'une configuration politique défavorable. En s'appuyant sur une enquête ethnographique, il analyse le processus de traduction opéré à partir des années 1990 par les membres d'un collectif de cyclistes. Occupant initialement une position marginale vis-à-vis des réseaux des partis politiques, ces agents parviennent progressivement à mettre à l'agenda de la " transition " vers une mobilité durable, en valorisant des ressources expertes et internationales. Par le biais de stratégies de professionnalisation et d'entrisme dans les administrations, ils impulsent le développement de modes de transport collectifs et peu polluants. Face aux contradictions persistantes de l'action publique en la matière, ils cherchent finalement à faire usage de leur insertion dans le champ politico-administratif pour garantir l'autonomie de leur contribution, par la mise en place d'un nouveau cadre légal tributaire des ressources spécifiques de l'expertise internationale. L'analyse de ce processus met en lumière la nécessité d'étudier les " trajectoires de transition " à l'aune des trajectoires sociales des agents qui les promeuvent. La " transition » vers une mobilité durable est ainsi conditionnée à Mexico par une transformation de l'espace social de production des politiques urbaines, marquée par l'émergence d'agents porteurs de légitimités nouvelles qui opèrent des déplacements et des échanges entre différents champs. Ces mouvements révèlent, davantage qu'une opposition entre capital politique local et expertise internationale, des logiques complexes de transfert entre ces deux types de ressources. 


\title{
Transition écologique et trajectoires sociales : les traducteurs de la mobilité durable à Mexico entre expertise internationale et politique locale
}

\author{
AUDREY CHÉRUBIN \\ Doctorante en sociologie sous contrat du \\ Centre national de la recherche scientifique (CNRS) \\ Centre de recherche et de documentation sur les Amériques \\ (CREDA, UMR 7229 CNRS - Paris 3), \\ Université Paris 3 - Sorbonne Nouvelle, USPC \\ Centre d'études mexicaines et centraméricaines \\ (CEMCA, UMIFRE 16 CNRS-MEAE)
}

Près de 34,5 millions de trajets s'effectuent chaque jour dans la Zone métropolitaine de la vallée de Mexico', dont plus de la moitié en véhicules motorisés : voitures individuelles, et taxis et bus souvent de faible capacité (les microbuses) bénéficiant de concessions individuelles de service public (INEGI, 2017). Ce trafic intense est le fruit d'un développement du transport basé depuis la première moitié du $20^{\mathrm{e}}$ siècle sur la construction d'infrastructures routières répondant et contribuant dans le même temps à l'expansion urbaine (Schteingart et Ibarra, 2016). II représente désormais la première cause locale de pollution atmosphérique de l'agglomération, qui figure parmi les plus polluées du globe. Face à l'urgence de la situation et en conformité avec les injonctions des organisations internationales, la ville de Mexico a entrepris, depuis le début des années 2000, une réorientation des politiques de transport urbain placée sous le signe officiel de la «transition» vers une mobilité durable, paradigme de gestion urbaine qui recouvre des vues divergentes autour d'un accord minimal sur l'enjeu de la préservation de l'environnement (Palmer, 2010 ; Scanu, 2014). Cette réorientation se traduit tout d'abord par l'expérimentation de nouveaux modes de transport, tels que le Metrobús, système de bus de grande capacité circulant sur des voies réservées (Bus

(CIien social et Politicutes no $82-2019$

ENGAGEMENTS ET ACTION PUBLIQUE FACE À LA CRISE CLIMATIQUE 
Rapid Transit) validé par I'ONU dans le cadre du Mécanisme de développement propre, et Ecobici, $1^{\text {er }}$ réseau de bicyclettes publiques d'Amérique latine. Elle est confortée en 2014 par la promulgation de la Loi de mobilité. Ce nouveau cadre légal impose une hiérarchie favorable aux piétons, aux cyclistes et au transport public au détriment des voitures. II vise à décourager les projets immobiliers dans les zones non couvertes par le réseau de transport public. II réorganise, par ailleurs, le service de transport public concédé pour en confier la gestion à un nombre limité d'entreprises opérant sous le contrôle d'un organisme public de régulation. Il cherche enfin à garantir l'ensemble de ces transformations par un processus élaboré de planification et de suivi basé sur l'expertise et la participation de la société civile. Remettant en cause la valorisation culturelle de l'automobile comme symbole d'appartenance aux classes moyennes et supérieures (Pérez López, 2013), ainsi que les relations établies depuis près d'un siècle (Davis, 1994) entre les pouvoirs publics et les acteurs économiques de la construction (David, 2013) et du transport concédé (Negrete, 2018), ces politiques se heurtent à de nombreuses résistances, qui freinent la marche vers la «transition». L'objet de cet article n'est pas de retracer ces conflits, mais de comprendre comment, dans ce contexte, la «transition » a cependant pu s'engager. Nous étudierons pour ce faire le processus de traduction (Callon, 1986; Hassenteufel et de Maillard, 2013) de la mobilité durable opéré par des agents locaux, en considérant qu'il est nécessaire d'étudier l'apparition de nouveaux secteurs de l'action publique en tenant compte des trajectoires de leurs promoteurs, de leurs ressources et positions dans différents champs (militant, professionnel, politico-administratif, etc.) (Dubois, 2014), et des conversions, entendues comme transpositions de dispositions et de compétences, qu'ils y opèrent (Tissot, Gaubert et Lechien, 2005).

En accord avec la méthode proposée par la sociologie de la traduction, le travail présenté ici s'attachera à un groupe d'agents en particulier, qu'il suivra tout au long du processus de réorientation des politiques publiques vers une mobilité durable. Le point de départ retenu se situe à la fin des années 1990, quand le mouvement cycliste de la ville de Mexico, après une première tentative d'organisation dans les années 1980, prend une nouvelle impulsion avec la création en 1998 de l'association Ciclomecas ${ }^{2}$. Nous nous centrerons sur trois des membres fondateurs du collectif, qui figurent, dès 
lors, parmi les plus actifs: Sofia, César, et Francisco. II ne s'agit pas pour autant d'attribuer à ces agents la responsabilité exclusive de la «transition » ni d'occulter l'existence de mouvements préalables en ce sens. Postulant qu'il est nécessaire pour comprendre comment s'opère le changement d'être attentif à la manière dont ils se positionnent relativement à d'autres protagonistes, nous ferons place, au fil du récit, à des agents de statuts divers, politicoadministratifs, économiques ou associatifs, locaux et internationaux. Par ailleurs, nous montrerons comment I'héritage des mouvements précédents a pu constituer une ressource pour ces militants. La focalisation sur un groupe ciblé permet cependant d'analyser de manière fine les multiples opérations par lesquelles se déploie sur le long terme la «transition». Le choix de Ciclomecas repose à cet égard sur l'ancienneté et sur la continuité de l'implication de ce groupe tout au long des vingt dernières années, période durant laquelle ont été opérées des avancées significatives en matière de mobilité durable.

Le présent article s'appuie principalement sur une série d'entretiens approfondis ${ }^{3}$, à la fois thématiques et biographiques, conduits avec les individus étudiés dans le but d'éclairer leurs actions, leurs interactions et leurs discours au regard de leurs trajectoires sociales (Demazière, 2011; Dubar, 1998). En complément de ces données, il mobilise également un corpus issu de sources documentaires diverses (administrative, experte, universitaire, journalistique et militante) relatif à ces agents et aux projets auxquels ils prennent part. L'analyse se fonde sur une enquête ethnographique menée depuis 2015, qui inclut des observations participantes des événements et des projets relatifs aux politiques de transport (congrès, forums ou célébrations, réunions de travail et ateliers) ainsi que des entretiens menés avec un ensemble plus large d'enquêtés retenus pour avoir joué un rôle important au cours des débats ${ }^{4}$.

À l'aide de ces éléments, nous verrons comment ces militants, qui occupent initialement une position marginale dans l'espace de production des politiques publiques, parviennent progressivement à mettre à l'agenda la «transition » vers une mobilité durable en valorisant des ressources expertes et internationales (1), se professionnalisent et se rapprochent de l'espace politico-administratif (2), et cherchent finalement à acquérir une relative autonomie vis-à-vis du jeu politique (3). 


\section{Des tours à vélo à la réalisation de la première piste cyclable : la valorisation progressive d'une expertise internationale}

\subsection{Les «utopistes 》 marginaux : des débuts politiques difficiles}

Appartenant aux classes moyennes urbaines cultivées de la génération des années 1970, les membres de Ciclomecas se forment dans un environnement politique marqué par la reconfiguration des rapports de pouvoirs et la recherche de nouveaux référentiels politiques après la déstabilisation du régime néocorporatiste du Parti révolutionnaire institutionnel (PRI), qui a dirigé le pays sans interruption de 1929 à 2000. Si leurs trajectoires militantes s'inscrivent dans une politisation à gauche de l'échiquier politique, héritée d'une sensibilité politique familiale, elles révèlent une relative marginalité visà-vis des réseaux des partis politiques ${ }^{5}$. Originaire de Mexico, Sofia arrive avec ses parents en 1977, à l'âge de sept ans, dans la ville voisine de Cuernavaca qui constitue à cette époque un lieu emblématique de la gauche radicale mexicaine et internationale, où convergent les militants de 1968, les émules de la théologie de la libération, les penseurs critiques de la théorie du développement et les réfugiés des dictatures chilienne, argentine et uruguayenne. Dès le début des années 1990, elle tente de s'investir dans la vie politique locale. Mais elle se heurte à la politique partisane, faisant prévaloir selon elle des intérêts privés. Elle se rapproche alors du mouvement zapatiste, dans lequel elle voit un modèle de démocratie directe.

César, fils d'un ingénieur et d'une sociologue tous deux enseignantschercheurs à Mexico, participe lui aussi aux réunions zapatistes. Dans les années 1990, il s'affilie au Parti de la révolution démocratique (PRD), qui s'impose comme le principal parti d'opposition de gauche au PRI et suscite un grand espoir en remportant les premières élections de la ville de Mexico en 1997. Mais il ne trouve pas sa place au sein d'une organisation dont il juge les pratiques trop verticales et qui favorise les savoirs de gouvernement au détriment des ressources militantes ayant présidé à sa construction (Combes, 2011). Au terme de la première année de gouvernement, il quitte le parti après près de six ans d'engagement, lassé d'être «le grinch des puppets» : «celui qui venait de l'extérieur, le critique de tous les processus ${ }^{6} \gg$.

Francisco grandit quant à lui au sein d'une famille de la gauche intellectuelle catholique de la capitale. Son père, architecte, contribue à des projets de 
logements sociaux tandis que sa mère, bibliothécaire, fréquente les tenants du «nouveau réalisme» mexicain. Lui-même côtoie les cercles militants étudiants de la fin des années 1980. S'il participe à la marche organisée en 1988 par Cuauhtémoc Cardenas, futur leader du PRD, pour contester sa défaite aux élections présidentielles, il ne s'engage pas dans la création du parti l'année suivante, préférant «rester critique ${ }^{7} »$. Dès 1998, il entre cependant en contact avec la nouvelle administration perrediste de Mexico afin d'exposer ses idées sur les politiques de la ville, mais son entreprise reste vaine.

En effet, si Ciclomecas se donne dès l'origine l'ambition d'influer sur les politiques publiques locales, les premières années de l'organisation sont à cet égard laborieuses. Les récits des militants expriment leur difficulté à être considérés comme des interlocuteurs crédibles par les responsables politiques:

Ça a été des années à se taper la tête contre le mur! On demandait des rendez-vous, on nous recevait et on nous disait « Hein? » ou «Félicitations les jeunes d'être si utopistes, mais ce que vous dîtes ne va jamais arriver». Ou : «Vous êtes kamikazes, si vous voulez faire de l'exercice allez faire des tours à Chapultepec [parc public]. » Ou alors on nous demandait : «Où sont tes troupes?» «Mais je n'ai pas de troupes de quoi que soit ! » Nous ne sommes pas des politiques tu sais, à répartir des prébendes ${ }^{8}[\ldots]$.

Les débuts du collectif correspondent certes à une délicate période de transition pour le PRD, parti-mouvement qui fait l'épreuve de sa première expérience de gouvernement, et la mobilité durable n'est pas la priorité de l'agenda politique. Mais la difficulté de ces militants tient également à leur extériorité vis-à-vis de réseaux d'échange politique (Vomarro et Combes, 2012) basés sur la redistribution ciblée à des bases populaires (《les troupes» auxquelles on offre des «prébendes »). Face à cette situation, l'activité de Ciclomecas réside essentiellement, dans un premier temps, dans l'organisation de tours à vélo et d'événements festifs visant à changer l'image de la bicyclette, utilisée à Mexico par les classes populaires et associée à la pauvreté?

\section{$1.2 \ll$ Regarde Portland 》 : la valorisation de l'expertise internationale}

Les membres du groupe disposent cependant dès l'origine d'autres ressources, qu'ils vont progressivement développer et valoriser pour se ménager une 
porte d'entrée dans la production des politiques publiques locales de mobilité. En effet, ils se distinguent par un haut degré de spécialisation sur les thématiques environnementales et/ou urbaines, et par la détention d'un ensemble de ressources que l'on peut regrouper sous le terme de «capital international » (Wagner et Réau, 2015) : la maîtrise de plusieurs langues, la connaissance des modèles et des formes du discours environnementaliste international ou la possession de réseaux de relations internationaux. Sofia, diplômée d'une licence en communication et d'une maîtrise en développement rural, commence une carrière de journaliste en couvrant l'actualité environnementale pour un groupe de presse de Cuernavaca. Elle part ensuite rejoindre sa sœur aînée à Montréal, où elle s'engage dans une station de radio bilingue anglais-espagnol et s'initie au cyclisme urbain. De retour au Mexique, ses compétences linguistiques lui permettent d'obtenir un emploi à mi-temps bien rémunéré comme assistante pour une chercheuse nord-américaine. Elle met alors à profit son temps libre pour suivre des formations complémentaires sur la gestion de l'environnement et sur l'élaboration des politiques publiques, et elle s'engage dans des associations écologistes. César découvre quant à lui le vélo en Allemagne, où il passe un an à l'occasion d'un congé sabbatique universitaire pris par ses parents. II débute ses études par une licence de physique, durant laquelle il travaille sur la pollution environnementale en milieu urbain et effectue un échange universitaire en Espagne. II s'oriente ensuite vers les sciences sociales dans le cadre d'une maîtrise en études urbaines d'une prestigieuse institution mexicaine. Francisco, enfin, devient un cycliste quotidien à Manchester, où il effectue une maîtrise en administration et mise en œuvre de projets de développement; il suit ensuite une maîtrise en ingénierie du transport spécialisée en développement durable aux Pays-Bas, en partenariat avec I'UNESCO.

Les «ciclomecas» acquièrent peu à peu des compétences de plus en plus spécifiques et étendent leurs réseaux internationaux. Collectivement ou individuellement, ils participent à des forums et à des congrès dédiés à la mobilité urbaine durable. Sofia explique comment cette spécialisation s'intègre à une stratégie visant à s'imposer dans la production des politiques publiques :

Nous avons un savoir supérieur aux hommes politiques sur ces thèmes, ce qui nous donne un avantage compétitif. On ne peut pas être contredits. C'est crucial d'avoir des données, d'avoir fait son travail et d'être aligné sur les bonnes pratiques internationales. Et donc quand ils viennent et te demandent, tu dis : «Regarde Portland, tatata, à New York 
je ne sais pas quoi...» Et eux : «C'est que je croyais... dans mon village...» «Tu croyais, tu pensais... Tes a priori, ton ignorance, ne peuvent pas faire la politique ${ }^{10} . »$

Pour que les hommes politiques «viennent et te demandent», encore faut-il cependant se faire connaître, et reconnaître. L'approfondissement des savoirs et savoir-faire n'est pas seulement technique; il se joue aussi sur le terrain de la communication. Maîtrisant parfaitement les outils électroniques, ce qui constitue alors un savoir-faire distinctif, les «ciclomecas» se dotent dès 1998 d'une page internet et d'un forum de discussion en ligne. Mais leur action d'autopromotion s'affine au fil de l'expérience qu'ils font des réseaux d'advocacy internationaux. Ils multiplient les conférences, interviennent dans les universités, investissent les médias, réalisent des actions «coup de poing ${ }^{11} »$. Suivant des stratégies de «double jeu » (Dezalay et Garth, 2001) par lesquelles ils font valoir, à l'international, leur connaissance «du terrain » et, localement, leur expérience internationale, ils se positionnent progressivement comme des passeurs de la mobilité urbaine durable entre, d'un côté, l'Europe et l'Amérique du Nord et, de l'autre, le Mexique et plus largement I'Amérique latine. Par le biais des contacts noués au Canada, Sofia rédige ainsi l'adaptation pour l'Amérique latine d'un guide pratique sur l'écologie urbaine, avec le soutien de la Commission européenne. Les «ciclomecas» reçoivent bientôt la visite de cyclistes venus du Chili et de la Colombie.

Cette stratégie est portée à son paroxysme en 2002, date à laquelle elle produit ses premiers effets notables. Ciclomecas est alors au plus fort d'une campagne de protestation contre le projet du nouveau maire perrediste de la ville, Andrés Manuel López Obrador, d'ajouter un second étage au périphérique autoroutier, le «Segundo Piso». Tandis que cette campagne, pour laquelle les «ciclomecas» mobilisent habilement les médias, permet au groupe de se faire connaître localement, Sofia présente dans des conférences internationales le plan d'aménagement d'une piste cyclable sur l'ancienne voie ferroviaire reliant Mexico à Cuernavaca. Elle est alors contactée par le Département de l'environnement de la mairie de Mexico, qui la charge de réaliser cette piste cyclable dans le cadre de la mesure légale de compensation environnementale associée au Segundo Piso. Dans le même temps, Francisco apprend, par le biais de ses contacts internationaux, la création à Mexico du Laboratoire de transport et durabilité, premier centre d'expertise locale du Global Institute for Environment, un think tank international effectuant des consultances auprès des décideurs politiques. Épaulé par les autres membres 
du groupe cycliste, il parvient à se faire embaucher par l'organisation, à partir de laquelle il œuvre notamment à la mise en place de la première ligne de Metrobús, inaugurée par la Direction de l'environnement en 2005. Lors de l'ouverture de la piste cyclable Mexico-Cuernavaca en 2004, c'est à lui que revient l'honneur de prononcer le discours, aux côtés du maire et en tant que représentant du Laboratoire de transport et durabilité. L'histoire du collectif entame un nouveau chapitre, celui du rapprochement avec le champ politico-administratif et de la répartition des rôles.

\section{Militants, consultants et fonctionnaires : la répartition des rôles}

\subsection{Militants et consultants : «bons flics/mauvais flics》}

Entrer au Laboratoire de transport et durabilité offre à Francisco l'occasion d'accumuler de nouvelles ressources, qui servent la dynamique de professionnalisation d'une partie du groupe cycliste. Cela lui permet en premier lieu de collaborer avec des consultants spécialisés qui ne sont pas issus du militantisme. C'est le cas de Maria Gálvez, récemment diplômée d'une maîtrise en gestion environnementale avec une spécialisation en transport et développement urbain d'une université de la Ivy League. Si la collaboration des deux consultants au sein du centre est brève, Maria Gálvez rejoignant l'équipe I'année du départ de Francisco, les liens qu'ils établissent alors perdurent et joueront par la suite un rôle décisif. Francisco a par ailleurs l'occasion de tisser des relations plus approfondies avec les responsables politico-administratifs et avec les acteurs économiques du transport à Mexico dans le cadre des négociations menées avec eux pour la mise en place du Metrobús. II se fait enfin connaître des financeurs internationaux et, en particulier, de la Fondation Emsworth, une fondation d'une grande famille d'entrepreneurs états-uniens qui est une donatrice régulière du Global Institute for Environment et qui soutient le Laboratoire de transport et durabilité depuis sa création. Or cette fondation est également donatrice d'une autre ONG internationale de consultance : le Transportation Expertise Center. Elle invite alors cette $O N G$ à rencontrer l'équipe du Laboratoire de transport et durabilité afin d'évaluer les possibilités de collaboration à Mexico des deux groupes. En 2006, le Transportation Expertise Center décide d'ouvrir une branche locale et Francisco se positionne pour en obtenir la direction. Bien que ce dernier ne dispose pas de l'expérience et des réseaux au gouvernement 
requis pour le poste, il finit par l'emporter grâce à la confiance établie lors des rencontres avec la Fondation Emsworth et grâce à la recommandation d'un ancien professeur de la maîtrise qu'il a effectuée à Delft, qui connaît personnellement le directeur général du Transportation Expertise Center. Francisco recrute alors son équipe parmi ses relations à Ciclomecas et au Laboratoire de transport et durabilité, incluant notamment, dès 2007, César. S'établit un partage informel de la consultance entre le nouvel institut, qui s'axe davantage sur la mobilité non motorisée, et le Laboratoire de transport et durabilité, plus spécialisé dans le transport public massif.

Cet événement entérine le processus de professionnalisation d'une partie des membres fondateurs de Ciclomecas, ce qui donne lieu à un partage des rôles entre militants et consultants. Sofia explique ainsi :

Moi, je suis restée à Ciclomecas, qui est comme le «bras armé » du Transportation Expertise Center. C'est très drôle parce que César nous a présentés dans une réunion en disant : «C'est Ciclomecas, c'est le bras armé du Transportation Expertise Center! » Et moi : «Ah oui? Moi, j'ai toujours pensé que le Transportation Expertise Center était le bras technique de Ciclomecas. » Parce que, vraiment, on se complète. Eux, ils ont, tu sais, la cravate, la chemise et la diplomatie et, nous, on est dans la rue, avec les tennis et en disant «Non! ». Donc cette combinaison donne beaucoup de pouvoir, et on a besoin les uns des autres, et on s'utilise pour faire pression sur des thèmes. Nous nous sommes spécialisés, ce sont les «bons flics» et «mauvais flics» qui jouent ensemble ${ }^{12}$.

Cette division des tâches ne va pas sans provoquer certaines tensions, comme le suggère l'échange rapporté ci-dessus. Sofia reconnaît avoir ressenti une certaine amertume lors de l'inauguration de la piste cyclable MexicoCuernavaca, quand un projet préparé par la «société civile» a finalement été présenté par un «cabinet d'experts». Certains membres de Ciclomecas rejettent d'ailleurs catégoriquement toute forme de coopération avec le gouvernement en place et refusent de ce fait de collaborer avec les consultants. Néanmoins, I'association y trouve rapidement un intérêt propre, en obtenant par le biais du Transportation Expertise Center des financements de la Fondation Emsworth pour mener ses campagnes. Surtout, à l'instar de Sofia, les principaux représentants du groupe, qui cherchent depuis l'origine à intervenir dans les politiques publiques, sont conscients de l'avantage en ce sens du passage à «la diplomatie ${ }^{13} »$. Est ainsi consolidé un rapprochement 
avec les pouvoirs publics, qui connaît rapidement une nouvelle étape avec l'entrée d'alliés des «ciclomecas » dans les administrations.

\subsection{De la consultance à la mairie : des alliés au gouvernement}

Le rapprochement des militants, et désormais consultants, avec la sphère politico-administrative connaît une étape décisive sous le gouvernement Ebrard (2006-2012), durant lequel la mobilité durable urbaine s'installe dans l'agenda politique local. Si ces avancées s'opèrent à la faveur de la visibilité qu'a acquise cet enjeu à l'échelle internationale, elles sont également permises par l'intégration d'alliés au sein de la Direction de l'environnement.

Les élections de 2006 sont à cet égard décisives. Tout d'abord, la victoire de Marcelo Ebrard constitue un atout de taille pour les militants cyclistes. En effet, elle représente un changement dans les rapports de force internes du PRD, avec le passage d'une gauche axée sur les politiques de redistribution à un courant mettant davantage l'accent sur la transformation des modes de vie. Par ailleurs, des liens ont été noués de longue date par le mouvement cycliste avec cette personnalité politique. La première rencontre a ainsi lieu dès les années 1980, quand ce qui est alors le Mouvement cycliste de Mexico décide d'offrir une bicyclette au régent de la ville dans une première tentative de rapprochement avec les pouvoirs publics. Si les militants ne parviennent pas à se faire recevoir en haut lieu, on leur envoie un «assistant $d^{\prime}$ assistant d'assistant ${ }^{14} \gg$ : Marcelo Ebrard, qui consent à les accompagner pour un tour à vélo. Le contact est établi et l'homme politique d'ascendance française, qui observe à Paris le développement de la mobilité cycliste et en pressent le potentiel politique, demande à Ciclomecas son soutien en 1999 dans la campagne qu'il mène au sein d'un parti minoritaire de gauche. L'association, alors soucieuse de maintenir son extériorité vis-à-vis des réseaux partisans, refuse cette offre. Néanmoins, cette nouvelle rencontre permet de maintenir une continuité des échanges. D'autre part, Sofia entretient des relations suivies avec une autre figure politique montante : Victoria Álvarez. Formée elle aussi à l'environnementalisme à Cuernavaca, Victoria Álvarez fonde une association promouvant la participation citoyenne au développement durable, avant de se lancer en politique. En 2006, au terme d'un mandat de députée indépendante à l'Assemblée législative de Mexico, elle se rapproche de Marcelo Ebrard. Afin d'acquérir une position forte dans la campagne électorale, elle invite Sofia à participer à létablissement d'une liste d'engagements sociaux et environnementaux à soumettre aux candidats. Les 
membres du Transportation Expertise Center et du Laboratoire de transport et durabilité se joignent à ce projet qui débouche, avec le soutien financier de la Fondation Emsworth, sur l'élaboration d'un programme de mobilité durable. Quand Marcelo Ebrard est élu, Victoria Álvarez obtient la responsabilité de la Direction de l'environnement et entame une collaboration étroite avec les militants-consultants. Cette collaboration est scellée en 2008 par l'entrée dans son cabinet de Maria Gálvez, restée proche de Francisco depuis leur rencontre au Laboratoire de transport et durabilité, et qui devient alors directrice de la stratégie de mobilité en bicyclette.

La Direction de l'environnement s'appuie ainsi sur les propositions élaborées par le mouvement cycliste au cours de la décennie précédente. Parmi elles figure la création d'un réseau de pistes cyclables basé sur un travail conjoint du Transportation Expertise Center et de Ciclomecas, dont la réalisation débute en 2010. La même année est inauguré Ecobici. Parallèlement, le Laboratoire de transport et durabilité est employé à la préparation de quatre lignes supplémentaires de Metrobús. En 2009, Ciclomecas met en place une procédure d'évaluation et de suivi de l'action publique : «On débattait avec Victoria et Maria de chaque proposition. Ça a été le germe de la politique publique comme on la veut ${ }^{15}$. $\gg$

Les politiques publiques en matière de mobilité, soumises à des intérêts multiples, restent néanmoins contradictoires. En 2008, le gouvernement de la ville de Mexico amorce ainsi la réalisation de la Supervía Poniente, une autoroute de 5,2 km destinée à relier l'anneau périphérique au quartier de Santa Fe, mégaprojet immobilier conçu par des alliés politiques de Marcelo Ebrard dans le but d'en faire un espace clé de la métropole globale (Pérez Negrete, 2010). Alors que ce projet suscite pendant plusieurs années un débat retentissant, les «ciclomecas», aux premières loges de la contestation, demandent à Victoria Álvarez de s'y opposer publiquement, ce qu'elle refuse. Les tensions montent et Victoria Álvarez rompt ses relations avec eux. Mais ces derniers disposent désormais d'un ancrage au sein de l'administration, et leur influence continue de s'exercer en sous-main au travers de Maria Gálvez :

Avec la Supervía, à la moitié du mandat, Victoria Álvarez a dit à son équipe de ne plus travailler avec nous. [...] Le programme de Ciclomecas et du Transportation Expertise Center a alors été : «Aider Victoria même si Victoria ne le veut pas! » [rires]. L'équipe de Victoria Álvarez, avec 
Maria, nous passait les infos et nous, on regardait, on commentait et on repassait l'info à l'équipe, sans le nom du Transportation Expertise Center ${ }^{16}$.

Mi-2010, Maria Gálvez finit cependant par quitter la Direction de l'environnement et rejoint le Transportation Expertise Center. Les militants-consultants, s'ils sont parvenus à se rapprocher des sphères politico-administratives, y occupent une position précaire. Pour garantir la «transition » vers une mobilité durable et, dans le même temps, leur contribution à la production des politiques publiques, ils vont dès lors chercher à les inscrire dans la loi. Ce qui, à la veille de nouvelles élections, exige de consolider et d'élargir les alliances établies avec le champ politique.

\section{De la «vision» à la loi : entrer dans le jeu politique pour s'en autonomiser}

\subsection{Les élections de 2012 : consolider et élargir les alliances dans le champ politique}

L'année 2012, durant laquelle ont lieu simultanément les élections du maire et celles de l'Assemblée législative de Mexico, et qui est marquée par un fort taux de renouvellement des équipes administratives, constitue un moment crucial pour les protagonistes de la mobilité durable.

En premier lieu, ces derniers scellent leur alliance avec le PRD, donné de nouveau favori. Rompant avec leur politique d'extériorité vis-à-vis du jeu partisan, ils mettent à profit les relations qu'ils ont forgées dans l'espace politico-administratif pour intervenir dès la campagne interne du parti. Ainsi, César, accompagnant un membre du PRD qu'il a connu lors de la mise en place d'un programme de valorisation de l'espace public, participe à l'un des premiers déjeuners où Miguel Mancera, le prochain maire, est proposé comme candidat. Grâce à ces liens précocement établis, les «ciclomecas» favorisent ensuite l'entrée de Maria Gálvez dans la campagne de Miguel Mancera :

On a dit à Maria : «Maria on s'y met! » Car le problème est que jamais on ne s'est impliqués dans la campagne, et si tu ne t'impliques pas dans la campagne, après c'est difficile d'être à l'intérieur et de prendre des décisions. Donc, on a fait en sorte que Maria soit impliquée dans la campagne. Et Maria est comme un virus, quand elle s'implique elle entre jusque dans la cuisine ${ }^{17}$ ! 
Maria Gálvez met alors à contribution les ressources expertes et internationales distinctives de ce groupe d'agents:

Maria fut comme notre «cheval de Troie». Elle se positionne parfaitement et fait campagne pour Mancera, elle va à des forums, etc. Elle a réussi à s'imposer grâce à son expérience, mais aussi en profitant toujours des occasions de créer des relations internationales. Par exemple, elle a une relation excellente avec Janette Sadik-Kahn [responsable du New York City Department of Transportation de 2007 à 2013]. Plus toutes ses bases techniques et son parcours de formation, et ce soutien international... Au premier débat entre les candidats, elle a amené Mancera sur le thème de l'espace public. Et là, la candidate du PAN [Parti action nationale, principal parti d'opposition de droite] n'était pas du tout au point ${ }^{18}$ !

Si le groupe de militants-consultants dispose désormais d'un «cheval de Troie » dans le camp du PRD, il ne néglige pas pour autant la constitution de nouvelles alliances en vue des élections législatives, en particulier justement à droite de l'échiquier politique, le PAN représentant le concurrent le plus sérieux du PRD dans la ville de Mexico :

Notre stratégie a toujours été d'aller avec tout le monde, car au final l'objectif est que tous votent en faveur de la loi. Notre stratégie a toujours été de minimiser les conflits politiques [...]. Donc, à n'importe quelle chose à laquelle on t'invite, tu y vas. C'est du PAN, du PRD, peu importe. Parce que plus tu accumules de choses où tu les as aidés, plus tu as de possibilités, quand tu as besoin d'eux, de pouvoir leur dire: «Aide-moi avec ça. » Parfois, ça provoque des frictions avec les partis de gauche, mais au final, ils comprennent; eux-mêmes, ils changent leurs alliances ${ }^{19}$.

Conscients de la nécessité de pouvoir présenter leurs objectifs de politiques publiques comme ceux d'un vaste panorama d'acteurs, les «ciclomecas» entreprennent un travail de mobilisation des alliés constitués au fil du temps parmi les experts, les acteurs internationaux, le secteur associatif local et les acteurs économiques du transport. Ils font alors appel, avec le soutien financier de la Fondation Emsworth, à une ONG mexicaine spécialisée dans l'accompagnement de dialogues multiacteurs, qui compte dans son équipe un ancien membre du Transportation Expertise Center et des relations nouées par Sofia au cours de sa trajectoire militante. Les liens des «ciclomecas » avec 
le monde académique leur permettent également de rallier au projet l'une des principales facultés d'ingénierie. Ils invitent, par ailleurs, comme représentants du secteur privé des transports, les dirigeants des entreprises auxquelles a été attribuée l'exploitation des lignes de Metrobús lors des négociations conduites par le Laboratoire de transport et durabilité. Ce processus aboutit finalement à la création de la plateforme «Des citoyens avec une vision», réunissant plus de 110 organisations et individus représentants du monde académique, de la société civile et de l'initiative privée dans le but affiché de construire une vision partagée sur la mobilité durable qui serve d'apport pour l'élaboration des politiques publiques. II ne s'agit pas cependant de débattre de la mobilité durable, mais d'offrir une vitrine à un discours déjà construit, partagé par des agents aux intérêts convergents. Les points de divergence entre les participants sont d'ailleurs volontairement écartés, l'objectif étant de faire valoir un consensus : «Le but, c'est de la com', avoir un agenda commun. Dans les détails sur lesquels on n'est pas d'accord, ce type de choses n'entre pas. Parce que, sinon, ça génère des problèmes, et ce que tu ne veux pas, ce sont des dissensions ${ }^{20}$.»

Les Accords pour la mobilité qui en résultent insistent sur la transformation du cadre normatif et en proposent les lignes directrices. Largement diffusés, ils participent à placer la mobilité durable à l'agenda de la campagne électorale.

Le thème est, en particulier, repris par Carla Barrera, candidate à l'Assemblée législative de Mexico pour le PAN, en qui le groupe trouve une nouvelle alliée. Ce rapprochement est facilité par le partage de dispositions similaires, qui constituent le support de relations interpersonnelles. Diplômée d'une maîtrise en direction stratégique et gestion de l'innovation cohabilitée par deux grandes universités espagnoles, elle jouit d'une formation internationale de haut niveau. Par ailleurs, si elle est alors membre du PAN, Carla Barrera est issue du secteur associatif, et son insertion dans le réseau partisan est précaire. Attirée par la campagne menée en faveur de la mobilité durable, elle se rapproche de Francisco, qui mène les discussions avec les candidats et, à travers lui, des militants et consultants, avec qui elle organise des réunions dès mai 2012.

En juillet 2012, Miguel Mancera est élu à la tête du gouvernement et Maria Gálvez est nommée directrice générale de la planification de la Direction des transports, tandis que Carla Barrera est élue députée à l'Assemblée législative de la ville et s'intègre à la Commission de transport. Il s'agit désormais pour les «ciclomecas» de transformer l'essai en imposant la loi à laquelle ils aspirent. 


\section{2 «Une stratégie d'échecs impeccable» : conjuguer les ressources}

Au lendemain des élections, le groupe de militants-consultants va alors mener une action concertée pour imposer la Loi de mobilité en mobilisant simultanément les multiples ressources dont il dispose désormais. Au sein de l'administration, Maria Gálvez continue de faire valoir son capital d'expertise international pour se rapprocher de Miguel Mancera, court-circuitant une hiérarchie qui valorise d'autres agents, notamment issus du syndicalisme :

Le responsable était plein de bonnes intentions. II vient du syndicat des transports électriques, il est très à gauche, mais il ne savait rien. Face à ça, Maria savait tout. Elle était de tous les colloques internationaux... Maria était «l'enfant chérie » de la Direction des transports, la véritable directrice. Elle était en contact direct avec Mancera ${ }^{21}$.

Profitant de cette position, Maria Gálvez s'attelle à l'élaboration d'une proposition de loi, en collaboration constante avec le Transportation Expertise Center, Ciclomecas, ainsi que le Laboratoire de transport et durabilité. Ensemble, ils formulent un projet sur la base des diverses productions de ces organisations, ainsi que des législations internationales existantes, en particulier de la Loi de mobilité de Catalogne et de la Loi de mobilité du Brésil.

La loi doit cependant être approuvée par l'Assemblée législative de Mexico. Or le PRD est divisé et Miguel Mancera n'a pas le soutien nécessaire dans ses propres rangs pour l'imposer. Grâce aux liens établis pendant la campagne, les promoteurs de la mobilité durable trouvent alors une autre «championne» à l'assemblée : Carla Barrera.

Maria Gálvez est la véritable maman de la loi, mais, en tant que fonctionnaire publique, elle ne pouvait pas l'imposer. Et c'est Carla Barrera qui devient la championne du projet à l'Assemblée. II fallait quelqu'un à l'intérieur de l'Assemblée pour pousser, négocier, parler avec les coordinateurs, etc. À cause des divisions au PRD, personne ne voulait s'en charger ${ }^{22}$.

Les réunions de travail menées par Carla Barrera avec le Transportation Expertise Center, Ciclomecas et le Laboratoire de transport et durabilité pendant la campagne trouvent ainsi leur prolongation en octobre 2012 avec la création du Conseil technique de mobilité, bientôt rejoint par 23 organisations nationales et internationales, dont l'ONU et la Commission de mobilité de la 
confédération patronale de la République mexicaine où siège le dirigeant de l'entreprise opérant la première ligne de Metrobús. Et grâce au travail mené en parallèle au sein de la Direction des transports de Mexico, le Conseil est en mesure d'émettre une proposition de loi dès février 2013, présentée le mois suivant à la Commission de transport. Face aux divisions des députés PRD, qui gèlent l'initiative, Carla Barrera commence alors à forger une alliance avec les partis minoritaires, en même temps qu'elle se rapproche du gouvernement. En novembre 2013, l'exécutif joint ses forces au groupe de députés mené par le PAN en déposant à son tour une initiative de loi.

La bataille passe enfin par la publicisation du débat, hors des enceintes des cabinets gouvernementaux et de l'assemblée. Les «bons flics» peuvent ici compter sur le «bras armé » que constitue Ciclomecas, sur l'expérience d'advocacy de l'association et sur l'audience qu'elle a acquise dans les milieux militants local et international.

Moi, je reviens quand il s'agit de rassembler les forces. Moi, je suis celle du mégaphone: «Alerte, cette loi est importante, venez!», pour pousser publiquement, convoquer la presse, activer les réseaux... «Les microbuses blabla... » Carla, Maria m'appelaient: «Hé Sofia, il m'est arrivé ça, appuie-moi ${ }^{23} . \gg$

En effet, le transport public concédé de taxis, bus et microbuses est voué à être réorganisé au profit d'une concentration du service au sein de grandes entreprises placées sous le contrôle d'un organisme de régulation. Mais cette restructuration se heurte à une forte opposition du secteur, organisé en une multitude de microentreprises qui constituent une source d'emplois importante pour les classes populaires de la capitale et qui négocient les règles de l'opération avec les pouvoirs publics par l'intermédiaire d'associations civiles.

Alors que les dirigeants de ces regroupements multiplient les démonstrations de force par le biais de manifestations et de blocages qui se répercutent sur les débats à l'assemblée, les militants cyclistes, numériquement plus faibles, répondent par des actions «coup de poing 》tès médiatiques. Le 28 février 2014, alors que le projet de loi tarde à être approuvé, ils profitent ainsi du Ciclotón qui se déroule dans la ville pour faire converger les participants et des organisations civiles vers la statue de l'Ange de I'Indépendance, place hautement symbolique de Mexico, afin de faire pression sur les élus. Sofia conclut: 
On ne leur a pas laissé de chance, on a fait une stratégie d'échecs impeccable. II te faut un pion, un cavalier, la reine, le roi... Tu as besoin de toutes ces ressources. On sous-estime souvent la valeur des réseaux de confiance, qui ont à voir avec ton accomplissement tout au long de ta vie. Peut-être que nous n'avons pas la plus grande capacité de mobilisation, mais nous avons une histoire sur près de vingt ans ${ }^{24}$.

La conjugaison de ces efforts porte en effet ses fruits : le 30 avril 2014, au terme d'un processus qui aura duré près de deux ans et mobilisé des ressources accumulées sur plus de quinze ans, l'Assemblée législative de Mexico approuve la Loi de mobilité, promulguée le 14 juillet par Miguel Mancera.

\section{Conclusion : La «transition écologique», un révélateur des logiques de transfert entre expertise internationale et capital politique local}

L'analyse du processus de traduction de la mobilité durable à Mexico met en lumière la nécessité d'étudier les «trajectoires de transition» à l'aune des trajectoires sociales des agents qui les promeuvent. Ainsi, la «transition» vers une mobilité durable est conditionnée à Mexico par une transformation de l'espace social de production des politiques urbaines marquée par l'émergence d'agents porteurs de légitimités nouvelles opérant des déplacements et des échanges entre différents champs (militant, professionnel, politico-administratif). Au terme de l'analyse, il apparaît que la participation à la fabrique des politiques publiques locales continue d'être assujettie à la détention de capital politique : c'est en effet par le biais du rapprochement qu'ils opèrent avec les sphères politico-administratives que les militants-consultants parviennent à implémenter les infrastructures, puis le cadre légal, conformes à leurs aspirations. Cependant, l'obtention de ce capital politique, dont ils étaient initialement privés ou, du moins, faiblement pourvus, s'appuie sur leur détention d'autres ressources, expertes et internationales. Grâce à ces dernières, ils concurrencent dans le champ politique des syndicalistes d'entreprises publiques ou des leaders de regroupements de concessionnaires du transport public, soit des agents dont la légitimité est liée à la capacité à se positionner comme intermédiaires. Par ce biais, les militants-consultants remettent en cause les logiques d'échange politique traditionnelles, en particulier avec des secteurs organisés des classes populaires. Autrement dit, davantage qu'une 
concurrence entre capital politique et expertise internationale, ce qui se donne à voir à ce stade est une tendance à la transformation des conditions d'acquisition du capital politique au profit des détenteurs de ressources expertes. Néanmoins, la dernière opération du processus retraduit ici, avec l'imposition de la Loi de mobilité, consiste à faire usage de cette insertion dans le champ politique pour s'en autonomiser. C'est-à-dire à mobiliser un capital politique acquis grâce à des ressources expertes pour garantir un accès à la production des politiques publiques qui soit strictement tributaire des ressources spécifiques de l'expertise internationale.

Il est trop tôt pour juger de la réussite de cette entreprise, le processus d'intégration-autonomisation des experts vis-à-vis du champ politique étant amené à se poursuivre. Malgré les avancées réalisées par le groupe de promoteurs de la mobilité durable, la portée de la «transition» dépendra des moyens financiers qui seront attribués à la mise en œuvre de la loi et de la mise en correspondance des politiques de planification urbaine avec les objectifs de mobilité durable. Elle sera également liée à la capacité des militantsconsultants à agir sur les règles du jeu non écrites de l'action publique, face au poids des relations de longue durée unissant les agents politiques et économiques. Or ces défis continuent de requérir l'insertion des militantsconsultants dans les sphères politico-administratives, laquelle reste fragile et ambivalente. Ainsi, Maria Gálvez s'est de nouveau vue évincée du gouvernement en 2016 à la suite d'accusations de favoritisme dans l'attribution d'un marché public, ce qui semble révéler, en même temps que la potentielle reproduction des logiques présidant à la conduite de l'action publique par ces nouveaux agents, l'exclusion de cette outsider par la classe politique. La victoire massive aux élections locales et nationales en juillet $2018 \mathrm{du}$ nouveau parti de gauche Movimiento de Regeneración Nacional semble cependant préfigurer une nouvelle étape décisive. Faisant campagne sur la lutte contre le clientélisme et la corruption, les dirigeants de ce parti se sont appuyés pour constituer leurs rangs sur des non-professionnels de la politique. Cela se traduit, pour le secteur qui concerne plus spécifiquement cette enquête, par la nomination à la tête de la Direction des transports d'un militant-consultant de la mobilité durable diplômé du Massachusetts Institute of Technology. Ce dernier affirme vouloir entériner durant son mandat la «transition» écologique des transports, en commençant par l'application pleine et entière de la Loi de mobilité, et imposer une conduite experte de l'action publique en la matière. 


\section{Bibliographie}

Bourdieu, Pierre. 2000. Les Structures sociales de l'économie. Seuil, Paris.

Callon, Michel. 1986. «Éléments pour une sociologie de la traduction. La domestication des coquilles Saint-Jacques et des marins-pêcheurs dans la baie de Saint-Brieuc », L'Année sociologique, $36: 169-208$.

Combes, Hélène. 2011. Faire parti : trajectoires de gauche au Mexique. Paris, Karthala.

David, Louise. 2013. «La production urbaine de Mexico : entre financiarisation et construction territoriale. Une analyse de l'insertion du réseau financier transnational dans les marchés d'immobilier d'entreprise». Thèse de doctorat en aménagement territorial, Université Paris-Est.

Davis, Diane E. 1994. Urban Leviathan. Mexico City in the Twentieth Century. Philadelphia, Temple University Press.

Dubar, Claude. 1998. «Trajectoires sociales et formes identitaires. Clarifications conceptuelles et méthodologiques», Sociétés contemporaines, 29, 1:73-85.

Dubois, Vincent. 2014. «L'action de l'État, produit et enjeu des rapports entre espaces sociaux», Actes de la recherche en sciences sociales. 1, 201-202 : 11-25.

Demazière, Didier. 2011. «L'entretien biographique et la saisie des interactions avec autrui », Recherches qualitatives. 30, 1: 88-110.

Dezalay, Yves et Bryant Garth. 2001. «La construction juridique d'une politique de notables. Le double jeu des praticiens du barreau indien sur le marché de la vertu civique», Genèses, 4 , $45: 69-90$.

Fillieule, Olivier et Danielle Tartakowsky. 2008. «La manifestation dans l'espace public», dans Olivier Fillieule et Danielle Tartakowsky (dir.). La manifestation. Paris, Presses de Sciences Po : 129-161.

Hassenteufel, Patrick et Jacques de Maillard. 2013. «Convergence, transferts et traduction. Les apports de la comparaison transnationale», Gouvernement et action publique, 3 : 377-393.

Instituto Nacional de Estadística y Geografía (INEGI). 2017. Encuesta Origen. Destino en hogares de la Zona Metropolitana del Valle de México. Mexico, INEGl.

Joshua, Florence. 2015. Anticapitalistes : une sociologie historique de l'engagement. La Découverte, Paris.

Negrete, María Eugenia. 2018. « Microbuses y taxis en la Ciudad de México. Cuestiones de gobernanza», dans Patrick Le Galès et Vicente Ugalde (dir.). Gobernando la Ciudad de México. Lo que se gobierna y no se gobierna en una gran metrópoli. Mexico, Colegio de México : 478-511.

Nonjon, Magali. 2005. «Professionnels de la participation : savoir gérer son image militante», Politix, 2, $70: 89-112$.

Palmer, James. 2010. «Stopping the unstoppable? A discursive-institutionalist analysis of renewable transport fuel policy », Environment and Planning C: Government and Policy, 28 : 992-1010.

Péchu, Cécile. 2001. «Les générations militantes à droit au logement», Revue française de science politique, 1, $51:$ 73-103.

Pérez López, Ruth. 2013. «El sistema de bicicletas públicas "Ecobici": del cambio modal al cambio social», Espacialidades, 3, 2 : 104-124. 
Pérez Negrete, Margarita. 2010. Santa Fe : ciudad, espacio y g/obalización. Puebla, UIA Puebla/ UIA México.

Scanu, Emiliano. 2014. «La construction sociale de la mobilité durable urbaine : les discours sur les enjeux du transport dans la ville de Québec», Recherches sociographiques, 55, 2 : 333-362.

Schteingart, Martha et Valentin Ibarra. 2016. Desarrollo urbano-ambiental y movilidad en la Ciudad de México. Mexico, El Colegio de México.

Tissot, Sylvie, Christophe Gaubert et Marie-Hélène Lechien. 2005. Les reconversions militantes. Limoges, Presses Universitaires de Limoges.

Vomarro, Gilles et Hélène Combes. 2012. «Relations clientélaires ou politisation : pour dépasser certaines limites de l'étude du clientélisme», Cahiers des Amériques latines, 69 : 17-35.

Wagner, Anne-Catherine et Bertrand Réau. 2015. «Le capital international : un outil d'analyse de la reconfiguration des rapports de domination», dans Johanna Siméant (dir.). Guide de l'enquête globale en sciences sociales. Paris, CNRS éditions : 35-48.

Wisler, Dominique. 1999. «Médias et action collective. La couverture de presse des manifestations publiques en Suisse », Revue française de sociologie, 40, 1: 121-138.

\section{Notes}

1 La métropolitaine de la vallée de Mexico réunit la ville de Mexico et son extension sur 60 municipalités des États de Mexico et de Hidalgo. Cette définition, fixée en 2005, correspond à la zone de conurbation de la capitale sans pour autant constituer un échelon de gouvernement, la Constitution du Mexique proscrivant l'établissement d'une autorité intermédiaire entre les municipalités et les États fédérés.

2 Par souci d'anonymisation, tous les noms des organisations mentionnées au fil du texte ont été modifiés, de même que les noms des personnes, à l'exception de ceux des maires successifs de la ville.

3 Huit entretiens longs (quinze heures au total) réalisés à Mexico de mars à juillet 2018.

4 Quarante entretiens ont été conduits depuis le début de l'enquête. Selon la méthodologie employée par Pierre Bourdieu dans son enquête sur le marché du logement, la détermination des agents «pertinents » a répondu à deux critères, institutionnel et réputationnel (Bourdieu, 2000).

5 Dans un contexte national certes très différent, on rejoint ici les analyses sur l'apparition de nouvelles générations de militants à la suite de la crise politique et économique des années 1980 en France, qui conduit à l'émergence de nouvelles utopies, comme l'écologie (Joshua, 2015), et à la différenciation d'un «champ militant» opposé au «champ politique» (Péchu, 2001). Mais, comme le souligne Cécile Péchu, ce processus d'autonomisation n'est ni univoque ni permanent, ainsi que nous le verrons dans la suite de cet article.

6 Entretien réalisé le 08/03/18

7 Entretien réalisé le 05/06/18.

8 Entretien réalisé le 06/03/18. 
9 Pour les foyers à faibles revenus ne disposant pas de voitures, la bicyclette constitue fréquemment une solution de rechange pour les trajets effectués sur de courtes distances, le transport public étant utilisé pour les distances plus longues. En effet, si $41 \%$ de l'ensemble des foyers de la Zone métropolitaine de la vallée de Mexico ont au moins une voiture, c'est le cas de seulement $26 \%$ des foyers dont les revenus sont inférieurs à trois salaires minimum (INEGI, 2017).

\section{Entretien réalisé le 06/03/18.}

11 La capacité de ces militants à mobiliser les médias doit être également rapprochée, au-delà de leurs compétences propres, de l'adéquation de ces compétences, des enjeux et des formes de leur mobilisation avec les valeurs professionnelles et les dispositions culturelles des journalistes (Wisler, 1999; Fillieule et Tartakowsky, 2008).

12 Entretien réalisé le 06/03/18.

$13 \mathrm{Au}$ cœur de ces controverses, on retrouve la tension entre militantisme et professionnalisation, analysée notamment par Magali Nonjon à propos des politiques de la ville en France. Mais tandis que l'auteure décrit les tensions existant entre «les militants d'origine» et de nouveaux professionnels de la participation aux profils plus techniques, conduisant les premiers à «valoriser leur étiquette militante tout en sachant la neutraliser quand cette dernière devient trop encombrante » (Nonjon, 2005 : 99), les nouveaux consultants sont ici issus d'un groupe de militants disposant dès l'origine de ressources expertes. Le «double jeu» entre militantisme et professionnalisme se traduit dès lors davantage par un partage des rôles. Il conviendrait d'analyser cette répartition entre militants et consultants au vu des ressources et des contraintes individuelles de chacun des membres du groupe. Le format de cet article ne permet pas d'approfondir l'analyse de ce point, celle-ci semble relever, entre autres facteurs, d'une division genrée du travail d'advocacy.

14 Sofia. Entretien réalisé le 06/03/18.

15 Sofia, entretien réalisé le 06/03/18.

16 César, entretien réalisé le 08/03/18.

17 César, entretien réalisé le 08/03/18.

18 Sofia, entretien réalisé le 09/03/18.

19 Francisco, entretien réalisé le 08/03/18.

20 César, entretien réalisé le 08/03/18.

21 César, entretien réalisé le 08/03/18.

22 Sofia, entretien réalisé le 09/03/18.

23 Sofia, entretien réalisé le 09/03/18.

24 Entretien réalisé le 09/03/18. 